Portland State University

PDXScholar

Mechanical and Materials Engineering Faculty

Publications and Presentations

\title{
Fifty-Plus-Year Postflight Analysis of First Fluid Experiment Aboard a Spacecraft
}

\author{
Mark M. Weislogel \\ Portland State University, weisloge@pdx.edu \\ Yongkang Chen \\ Portland State University \\ William J. Masica \\ NASA Glenn Research Center \\ Fred J. Kohl \\ NASA Glenn Research Center \\ Robert D. Green \\ NASA Glenn Research Center
}

Follow this and additional works at: https://pdxscholar.library.pdx.edu/mengin_fac

Part of the Aerospace Engineering Commons, Materials Science and Engineering Commons, and the Mechanical Engineering Commons

Let us know how access to this document benefits you.

\section{Citation Details}

Weislogel, M. M., Chen, Y., Masica, W. J., Kohl, F. J., \& Green, R. D. (2017). Fifty-Plus-Year Postflight Analysis of First Fluid Experiment Aboard a Spacecraft. AIAA Journal.

This Article is brought to you for free and open access. It has been accepted for inclusion in Mechanical and Materials Engineering Faculty Publications and Presentations by an authorized administrator of PDXScholar. Please contact us if we can make this document more accessible: pdxscholar@pdx.edu. 


\title{
Fifty-Plus-Year Postflight Analysis of First Fluid Experiment Aboard a Spacecraft
}

\author{
Mark M. Weislogel* and Yongkang Chen \\ Portland State University Portland, Oregon 97102 \\ and

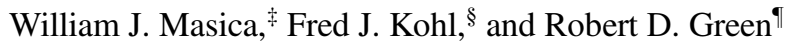 \\ NASA Glenn Research Center Cleveland, Ohio 44135
}

\section{DOI: $\underline{10.2514 / 1 . J 055484}$}

\begin{abstract}
This year marks the 55th anniversary of the first fluid physics experiment performed aboard a spacecraft during the Mercury-Atlas 7 mission. Since then, NASA has conducted over 80 fluids physics experiments aboard a variety of spacecraft, many of which have enhanced the understanding of large-length-scale capillary phenomena relevant to liquid management in the weightless state. As both celebration and demonstration, the Mercury-Atlas 7 fluids experiment is revisited in light of the current understanding of large-length-scale capillary fluidics. Employing a modern numerical tool, a rich variety of experimental outcomes are discovered that were not observed during the flight experiment. Interestingly, experimental support for these "newly computed" outcomes draws from 54-year-old drop-tower data collected by the original NASA investigator team. A direction forward for advanced tankage design is highlighted in summary.
\end{abstract}

\section{Nomenclature}

$a=$ semimajor axis of a hyperbola

$b=$ semiminor axis of a hyperbola

Bo $=$ Bond number; $\rho g R^{2} / \sigma$

$d=$ standpipe thickness (dimensionless)

$E_{r} \quad=\quad$ reduced surface energy

$g=$ acceleration field amplitude, $\mathrm{m} / \mathrm{s}^{2}$

$H=$ dimensionless free surface mean curvature

$l=$ dimensionless standpipe cylinder length

$l^{\prime}=$ dimensionless standpipe upper rim location

$R=$ uniform spherical tank of radius

$R_{\text {com }}=$ dimensionless radial coordinate of center of mass

$x$

$x$

dimensionless cylinder radius

transverse coordinate

axial coordinate

transverse coordinate

transverse coordinate

axial coordinate

dimensionless cylinder axis offset

contact angle, rad or deg

liquid fill fraction

fill fraction lower bound (unstable below) outer wall at the midplane wall at upper outer rim

$=$ fill fraction upper bound (unstable above)

$=$ liquid density, $\mathrm{kg} / \mathrm{m}^{3}$

$=$ surface tension, $\mathrm{N} / \mathrm{m}$

$=$ spherical polar angle for $g$-orientation

$=$ spherical azimuthal angle for $g$-orientation standpipe polar (tilt) angle with respect to tank z-axis

fill fraction where nodoid surfaces meet the standpipe

$=\quad$ fill fraction where nodoid surfaces meet standpipe outer

Received 6 July 2016; revision received 10 May 2017; accepted for publication 24 May 2017; published online 27 July 2017. Copyright @ 2017 by the American Institute of Aeronautics and Astronautics, Inc. All rights reserved. All requests for copying and permission to reprint should be submitted to CCC at www.copyright.com; employ the ISSN 0001-1452 (print) or 1533-385X (online) to initiate your request. See also AIAA Rights and Permissions www.aiaa.org/randp.

*Professor, Mechanical Engineering.

${ }^{\dagger}$ Research Faculty, Mechanical Engineering.

${ }^{\ddagger}$ Chief of Space Experiments Division, Retired.

${ }^{\S}$ Fluid Physics Program Manager, Retired.

${ }^{\top}$ Research Scientist.

\section{Introduction}

$\mathbf{F}$ IFTY-FIVE years ago, on 24 May 1962, the seventh MercuryAtlas (MA7) mission launched astronaut Scott Carpenter into an approximately $4 \mathrm{~h}$ orbit of the Earth. Adjacent to the right side of his headrest was mounted the first capillary fluidics experiment to be conducted in space. A video still image of this arrangement is shown in Fig. 1, with an original photo and schematic of the experiment apparatus shown in Fig. 2 and critical dimensions and properties provided in Table 1 . The video still image of the nearly weightless configuration of the fluid interface in Fig. $1 \mathrm{~b}$ compares well with historic NASA $2.2 \mathrm{~s}$ drop-tower test results using a scale model of the flight experiment shown inset.

The experiment was led by NASA Lewis Research Center engineers Petrash et al. [1] with conceptual input from Reynolds of Stanford University [2]. The purpose was to demonstrate the passive control of fluid interfaces in a weightless state by exploiting the effects of surface tension forces, container geometry, and liquid wetting. The experiment, hereafter referred to also as MA7, consisted of a spherical glass container with a wall-mounted right circular cylindrical baffle called a "standpipe". The spherical $83.8 \mathrm{~mm}$ i.d. $300 \mathrm{ml}$ chamber was partially filled to $20 \%$ with an assumed perfectly wetting dyed aqueous solution [1]. Three circumferential circular perforations at the base of the $48.3 \mathrm{~mm}$ long, $27.9 \mathrm{~mm}$ i.d., unrecorded o.d. standpipe permitted pressure communication between continuous fluid inside and outside of the cylinder. The standpipe serves as a low capillary pressure component that draws liquid inward providing a passive means of fluid positioning such that in the "low- $g$ " state the liquid could be located over the liquid exit port and the gas over the gas exit port, as suggested in Fig. 2. The drop-tower test images of Fig. 3 are the originals presented by Petrash et al. [1] in 1963. These images show that, for a variety of fill fractions and initial fluid orientations with respect to gravity, the fluid reoriented toward a symmetric configuration during freefall, with liquid filling the standpipe centered over an envisioned liquid exit port. It was such preflight experimental results that led to the subsequent selection of the particular dimensions and liquid fill fraction for the MA7 experiment.

Passive no-moving-parts fluid control as exhibited in Figs. $\underline{1 b}$ and $\underline{3}$ is desirable for numerous fluids management operations aboard spacecraft including propellant management, cryogen storage and handling, two-phase thermal control systems, and various lifesupport systems that include the processing of water. The simple MA7 experiment was conceived with primarily liquid propellant management in mind. Its successful demonstration added confidence to design engineers in the emerging field of large-length-scale capillary fluidics aboard spacecraft. 

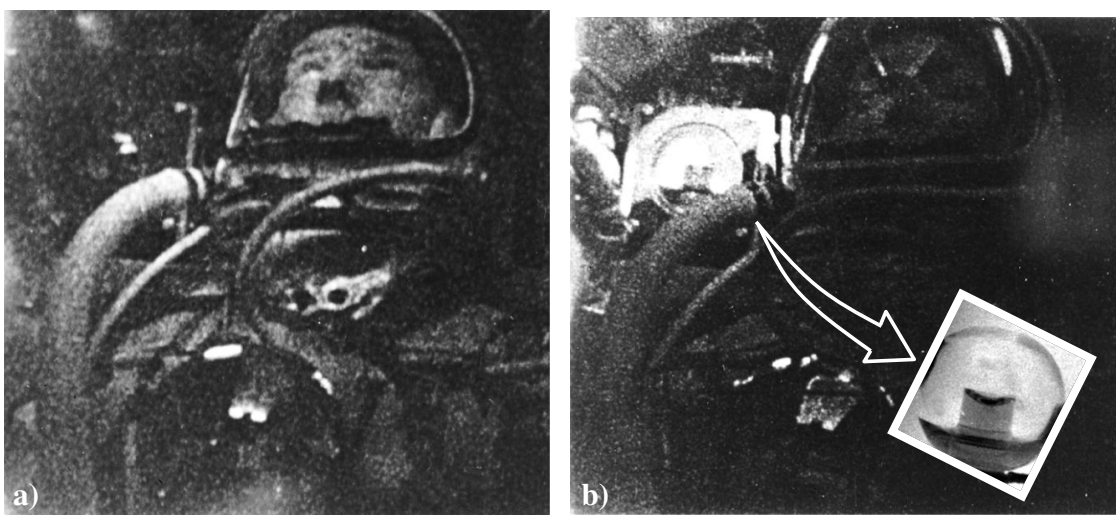

Fig. 1 a) Astronaut Scott Carpenter during MA7 flight with experiment to the left of Carpenter's head. b) Inset provides anticipated low- $g$ interface configuration observed during drop tower experiments of scale model. (Courtesy of NASA GRC.)

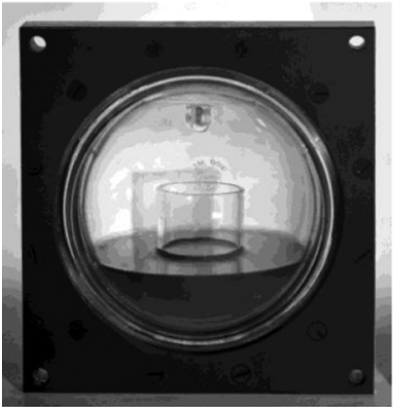

a)

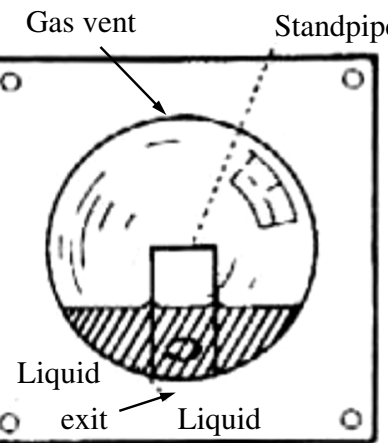

Front view

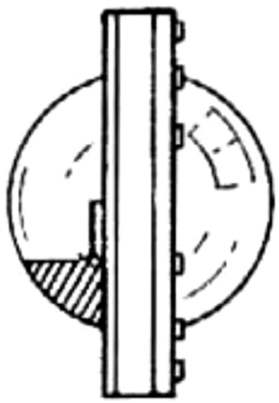

Side view

Fig. 2 a) Original photo of the MA7 capillary fluids experiment with flight fluid fill fraction. b) Views of experiment with conceptual liquid and gas exit ports identified. (Courtesy of NASA GRC).

Since 1962, in addition to many thousands of tests conducted in drop towers, low- $g$ aircraft, and $1 g$ laboratories, NASA has conducted numerous capillary fluids experiments in space aboard Apollo, Skylab, Shuttle, the Russian Mir Space Station, and currently the International Space Station (ISS). Concerning in-space research, two experiments are of particular relevance to MA7: the Interface Configuration Experiments performed on the Space Shuttle and Mir $[\underline{3}, \underline{4}]$ and the Capillary Flow Experiments performed on ISS [5,6]. The two salient results of these experiments are that 1) multiple nonaxisymmetric fluid interfaces can form in axially symmetric containers, and 2) slight container asymmetries can lead to large asymmetric shifts in the liquid configuration. An awareness of such possibilities leads one immediately to suspect that the MA7 experiment might exhibit strong asymmetric fluid interface tendencies as well.

In light of such possibilities, the MA7 geometry is revisited herein using a modern open-source numerical free-surface solver called SE-FIT [7]. SE-FIT employs Brakke's Surface Evolver (SE) program [8] as a kernel within a fluid interface tool (FIT) that improves the efficiency of conducting free surface equilibrium computations. One such improvement is the growing list of highly alterable prebuilt geometries

Table 1 Critical dimension and properties for MA7 experiment

\begin{tabular}{lc}
\hline \hline Quantity & Value \\
\hline Spherical tank i.d. & $83.8 \mathrm{~mm}$ \\
Standpipe tube i.d. & $27.9 \mathrm{~mm}$ \\
Standpipe tube o.d. & -- \\
Standpipe height & $48.3 \mathrm{~mm}$ \\
MA7 container material & Glass \\
Dyed water surface tension & $0.034 \mathrm{~N} / \mathrm{m}$ \\
Dyed water density & $1000 \mathrm{~kg} / \mathrm{m}^{3}$ \\
Dyed water/glass contact angle & $\sim 0 \mathrm{deg}$ \\
\hline \hline
\end{tabular}

included with the SE-FIT code. When selecting from these geometries, the user can modify a specific geometry and productively compute interface configurations with minimal training and no programming. This is now possible for the MA7 geometry. A sketch of such is provided in Fig. 4a, which includes a uniform spherical tank of radius $R$, standpipe of thickness $d$ with cylinder radius $r$, cylinder length $l$, cylinder axis offset $\delta$, contact angle $\theta$, surface tension $\sigma$, liquid density $\rho$, and acceleration field amplitude $g$ through Bond number $B o=\rho g R^{2} / \sigma$, which also includes $g$-direction through polar $\phi$ and azimuthal $\psi$ orientation angles. Nondimensionalizing all lengths by the tank radius $R$, for the $20 \%$ fluid fill fraction employed by MA7 and $B o=0$, the 50-plus-year postflight SE-FIT numerical predictions are presented in Figs. 3b-3d, which agree well with both flight experiments and droptower tests (Figs. $1 \mathrm{~b}$ and 3 , respectively).

As shown in Fig. 4, one of the numerical methods employed herein is called the contact line method and models the contact line on the spherical wall and on the inner and outer standpipe walls. A second approach models the interface between the gas and the spherical wall and will be referred to as the hybrid surface method. The methods are not elaborated herein.

\section{Cursory Numerical Study of Mercury-Atlas 7}

Stemming from our interest, concern, and exposure to asymmetric equilibrium surfaces in allegedly symmetric containers [3-6], at first glance, we suspect that the MA7 container is of the type that will yield asymmetric surfaces. In anticipation of such an occurrence, a dimensionless standpipe offset parameter $\delta$ is introduced into the SEFIT prebuilt model to assist in the investigation (see Fig. 4a). As a quick demonstration, in Fig. 5b, for a 50\% fill fraction, a symmetric equilibrium surface is computed in the MA7 container. As a means of perturbing the container geometry, as shown in Fig. 5c, when the standpipe is shifted $\delta=0.01$ (1\% of $R$ ), an asymmetric shift in the fluid configuration is computed. The standpipe is then displaced back 


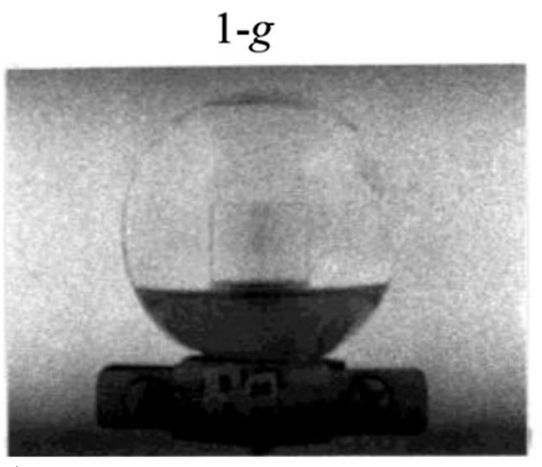

a)

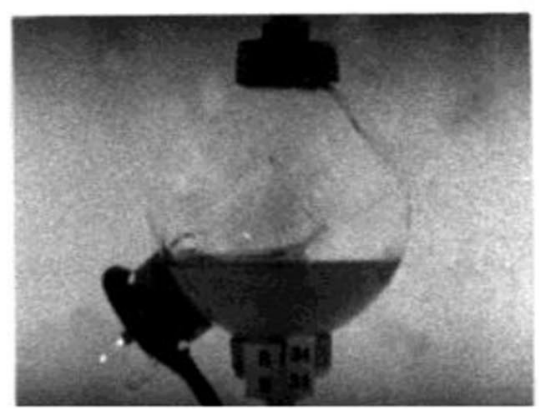

b)

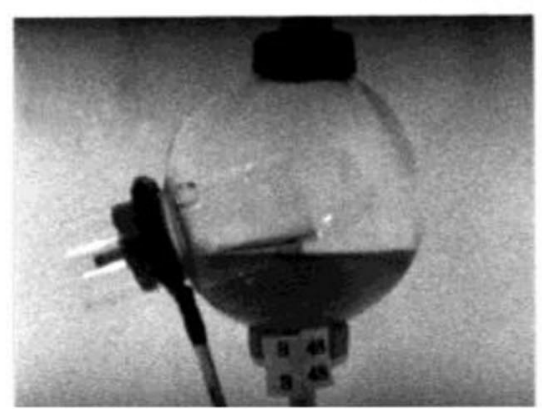

c)

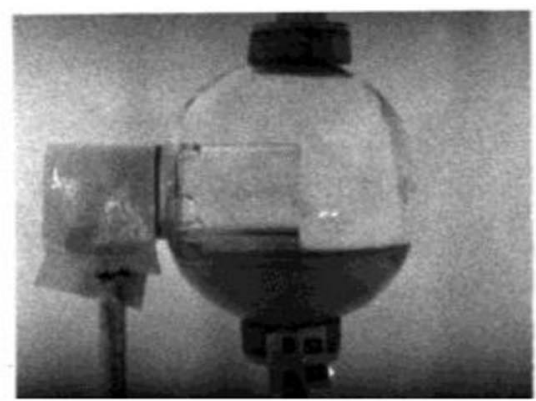

d)

Fig. 3 Original NASA drop tower experiments for MA7 scale model. Container tilt varied to demonstrate recoverable low- $g$ interface configuration for $20 \%$ fill fraction: a) $0 \mathrm{deg}$, b) $45 \mathrm{deg}$, c) $75 \mathrm{deg}$, and d) $90 \mathrm{deg}$. (Courtesy of NASA GRC.)

(recentered) to the center location $(\delta=0)$ and the surface recomputed to find the solution shown in Fig. 5d. This surface remains asymmetric with slightly lower energy than the symmetric interface of Fig. $\underline{5 b}$, despite the obvious shift in mass center. Thus, given enough time [4], it is certain that an asymmetric interface is preferred for an important range of tank standpipe geometries and fill fractions. In this case, the asymmetric surface is discovered via a geometric perturbation. With other methods such as the hybrid surface method, the asymmetric surfaces may also be obtained by perturbing the center of mass of the gas phase.

It will be demonstrated numerically that MA7 employed a fill fraction where such asymmetries do not arise. However, following a more thorough search of the NASA literature, it was found that additional drop-tower experiments performed at higher fill fractions and longer
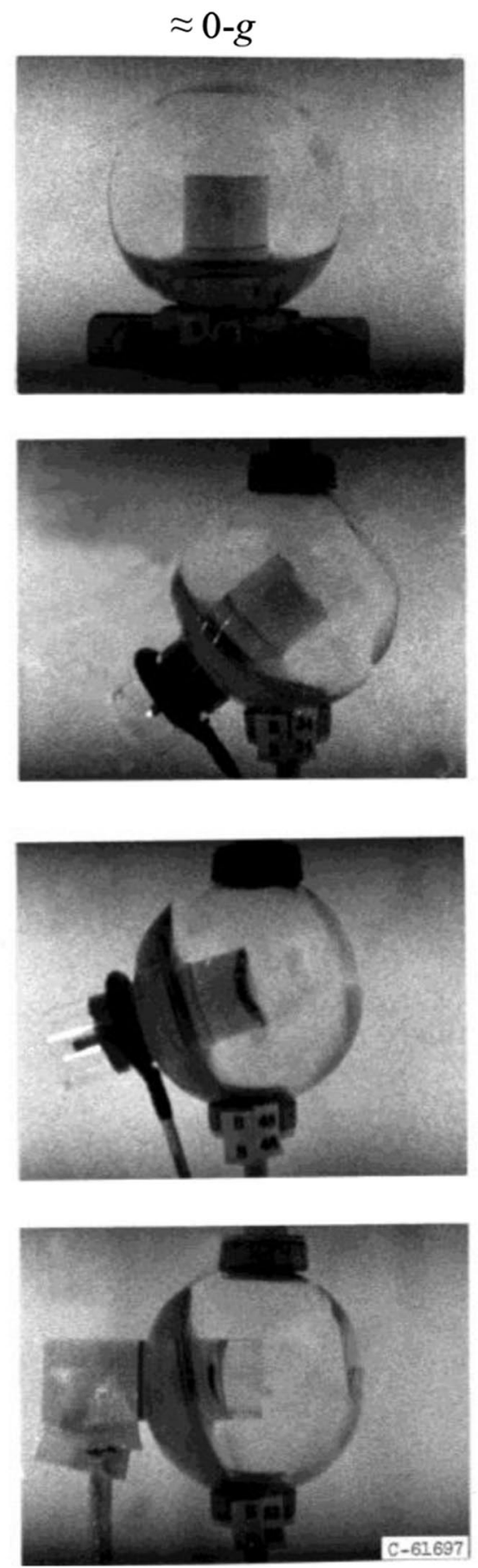

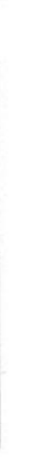



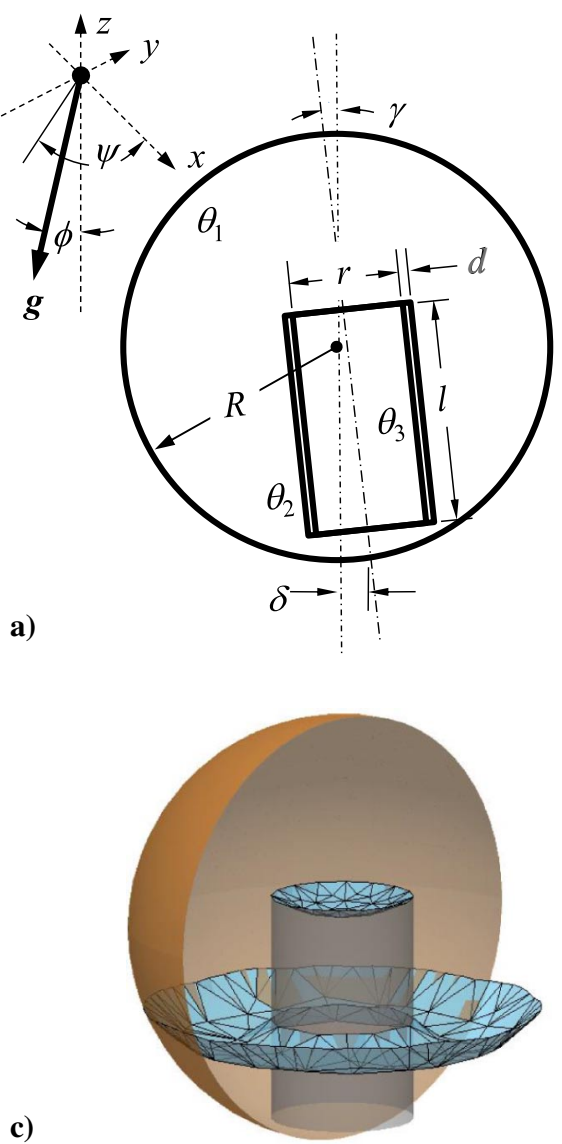

c)

Fig. 4 SE-FIT MA7 prebuilt model with $20 \%$ fill fraction: a) numerical geometry schematic, b) initial condition with $g=\infty(\phi=0, \psi=\pi / 2)$, c) roughly converged symmetric interface profile with d) high resolution deeply converged solution.

and for brevity in this presentation, only the standpipe radius $r$, length $l$, and fill fraction $\lambda$ are varied, with the remainder of properties held constant at $R=1, d=0, \delta=0, B o=0, \phi=0 \mathrm{deg}, \psi=90 \mathrm{deg}$, and for the MA7-dyed water, $\rho=1000 \mathrm{~kg} / \mathrm{m}^{3}, \sigma=0.034 \mathrm{~N} / \mathrm{m}$, and $\theta_{i}=0 \mathrm{deg}$. We note that when $B o=0$, it is superfluous to specify $\rho$ and $\sigma$ and that from this point forward all computations are performed for $B o=0$. However, because background accelerations are never zero in practice, specific values of $\rho$ and $\sigma$ are required to establish $B o \ll 1$. We recall that all lengths $d, \delta, l$, and $r$ are nondimensionalized by the tank radius such that $R=1$ in all SE-FIT computations presented in this paper.

A sample of possible equilibrium surfaces in the generalized tank is shown in Fig. 7. Extensive computations are readily performed using the PSF allowing the geometric conditions for each interface type to be identified. Further exhaustive computations are possible that consider multiple unconnected interfaces as well as finer geometric details such as a finite thickness standpipe, variable contact angle, nonconstant tank radius $R$, and others. The free surface configurations of Figs. $7 \mathrm{f}$ and $7 \mathrm{~g}$ are reminiscent of those for the ullage bubble formed in the spin-stabilized Gravity Probe B spacecraft as anticipated and computed by others [10-12]. The cryogenic liquid tank of Gravity Probe B is similar to $\overline{\mathrm{MA}}$ 7 but includes a cylindrical section connecting two spheroidal lids and an effective impenetrable cylindrical standpipe that extends from base to lid. In the MA7 container, the torus-shaped bubble is confined between the standpipe and spherical tank walls due only to wetting, geometry, and fill fraction, whereas in the Gravity Probe B tank, the torus-shaped ullage is also impacted by the added complexity of centripetal acceleration. Collicott [13] identifies asymmetric interface configurations in a spherical tank with a central vane structure for a specific range of fill fractions when $g=0$.

A selection of computed images illustrating surface configurations at different fill fractions is displayed in Fig. $\underline{8}$ for a case where

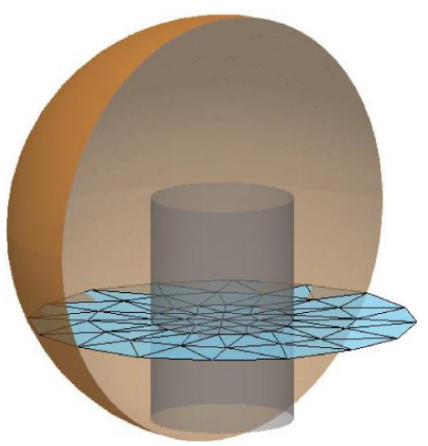

b)

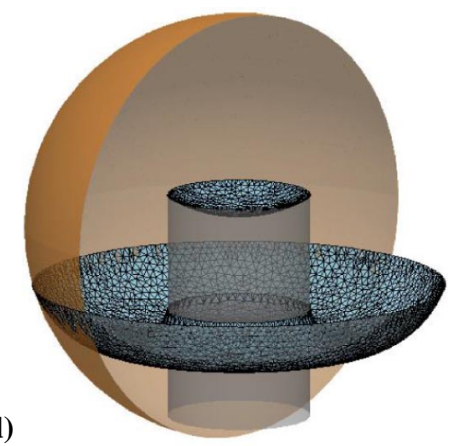




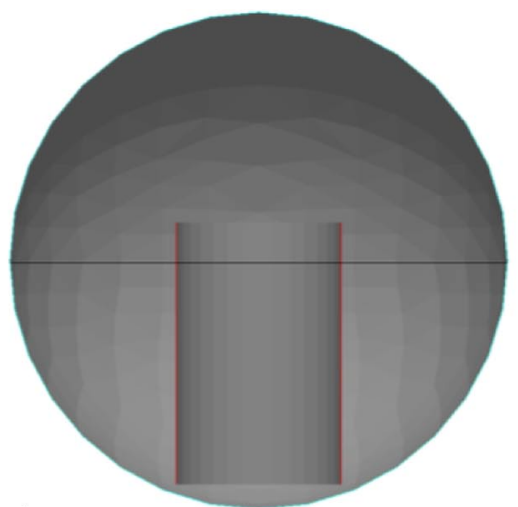

a)

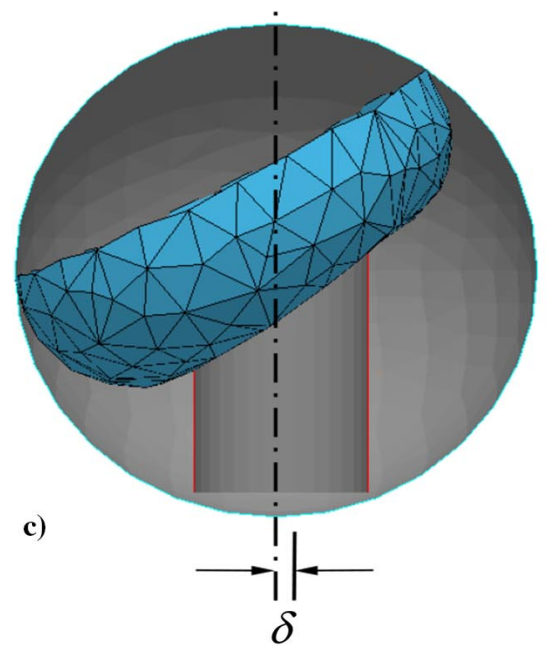

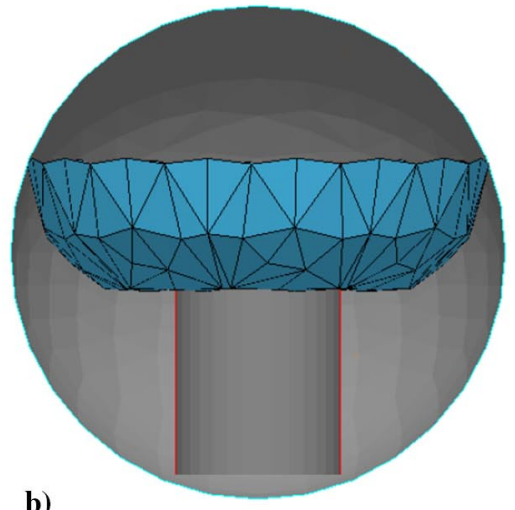

b)

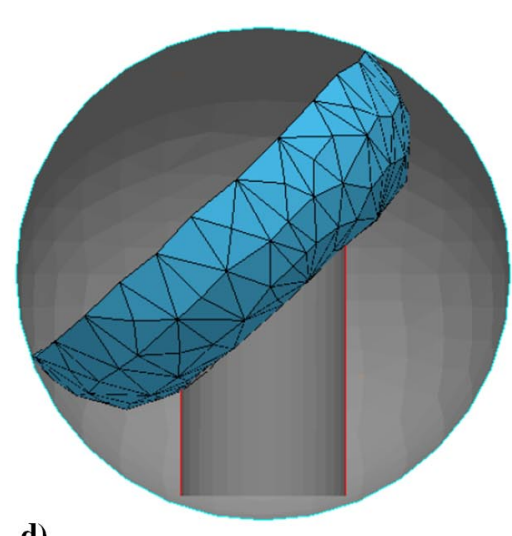

d)

fill fraction: a) Initial interface, $g=\infty, \delta=0$, b) symmetric, $g=0$,
1.53, and d) asymmetric with offset displaced back $\delta=0, g=0$,

Fig. 5 Computations of MA7 asymmetric equilibrium interface configuration, 50\% fill fraction: a) Initial interface, $g=\infty, \delta=0$, b) symmetric, $g=0$,
$\delta=0, E_{r}=-1.15$, c) asymmetric with standpipe offet $\delta=0.01, g=0, E_{r}=-1.53$, and d) asymmetric with offset displaced back $\delta=0, g=0$, $E_{r}=-1.52$.

Upon further inspection of Fig. 11, for the special case of $r=0.5$, it is found that, for the fill fraction range $0.3 \leq \lambda \leq 0.39$ and $0.54 \leq \lambda \leq 0.68$, the mean curvature $H$ of the free surface remains constant, leading to $E_{r} \sim \lambda$. Within the former range, the meniscus inside the standpipe actually moves downward with the increase of fill fraction, and at $\lambda=0.39$, the standpipe meniscus comes in contact with and establishes a "dry" region on the spherical tank wall. Within the latter fill fraction range, at $\lambda=0.54$, and with increasing fill fraction, the meniscus inside the standpipe begins to detach from the spherical wall and rise toward the standpipe upper inner rim. Because the contact angle is $0 \mathrm{deg}$ and $r=0.5$, the mean curvature of the surface within these two fill fraction ranges is exactly $1 / r=2$, which is twice that of the spherical wall mean curvature, $1 / R=1$. Despite identical curvature, the free surface inside the standpipe is spherical, whereas the free surface shape between the standpipe and the tank wall varies with the fill fraction. The nearly linear behavior of the reduced energy plot in the range $0.39<\lambda<0.54$ shown in Fig. 11 indicates a weak influence of the overall geometry on the surface energy as the standpipe simply fills with liquid.

For each standpipe radius, the range of fill fraction that produces asymmetric surfaces is approximated by the shaded region in Fig. 12. Note that a significant shift of the asymmetric surface conditions occurs in the vicinity of $r=0.5$. In the fill fraction range for asymmetric surfaces, it is observed that, for $r>0.5$, the meniscus inside the standpipe resides near the lower inner rim, partially exposing the tank wall within the standpipe. The meniscus inside the standpipe resides at the upper inner rim for $r<0.5$; see Fig. 10. A nearly empty standpipe for $r>0.5$ implies that the volume of the fluid is displaced outside of the standpipe, raising the level of the free surface to the point that asymmetric configurations are stable. The fill fraction range for the asymmetric surfaces reduces somewhat with increasing standpipe radius.

Such readily accessible computations identify fill fraction lower $\lambda_{l}$ and upper $\lambda_{u}$ bounds between which symmetric surfaces are unstable. The computational results reveal that, at the fill fraction lower bound, the contact line of the symmetric surface on the standpipe exterior wall reaches the horizontal tank midplane. It also reveals that, at the fill fraction upper bound, the contact line of the symmetric surface reaches the standpipe upper outer rim.

Furthermore, as displayed in Fig. 13, it is found that the free surface curvature, like the reduced surface energy (refer to Fig. 11), varies only slightly between unstable symmetric and stable asymmetric surfaces with all else equal. However, as suggested especially in Figs. $\underline{9}$ and 10 , the impact of the displacement of the liquid center of mass for the asymmetric surface is obvious and could have significant consequences in practice. As presented in Fig. 14, for fixed standpipe height $l^{\prime}=1.152$, for all the pipe radii studied, the radial shift of the liquid center of mass varies up to approximately $25 \%$ of the tank radius and up to $\approx 21 \%$ for the MA7 geometry $(r=0.333$, Fig. 14). Figure 14 also provides the range of fill fractions $\lambda$ that produce the axisymmetric-over-exit configuration for these conditions. Larger center-of-mass shifts are expected for longer standpipe lengths $l$.

\section{Further Symmetric Equilibrium Stability Considerations}

As similarly found by others, and specifically for cylindrical annular containers $[16,17]$, the symmetric surfaces within the MA7 tank geometry are nodoids produced by rotating a nodary about its axis. In this case, the nodary can be written parametrically as 
$1-g$

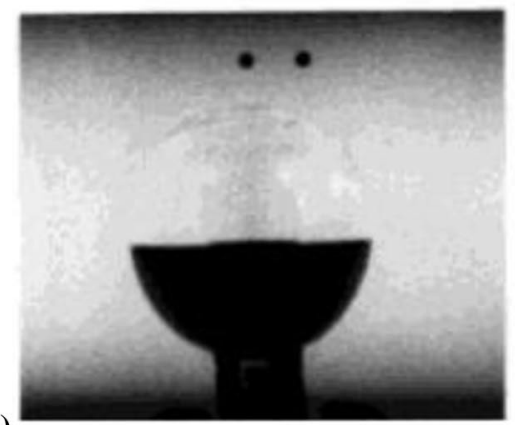

a)

b)

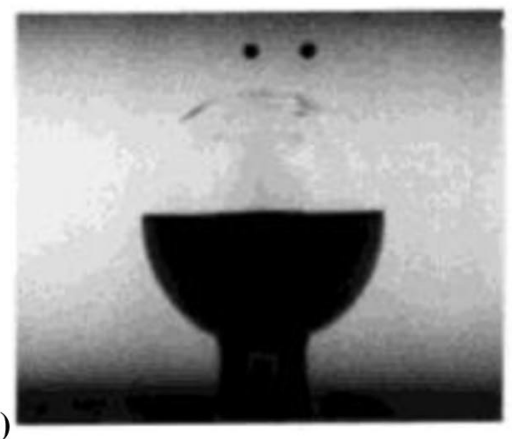

c)

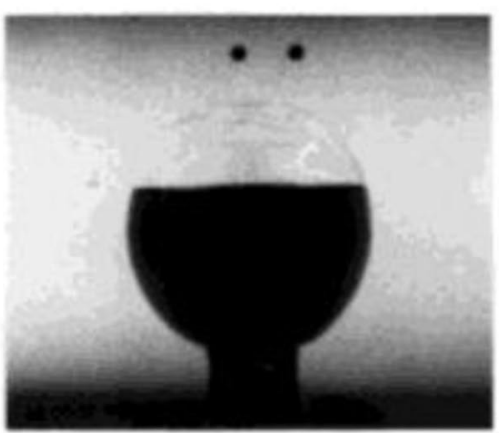

d)

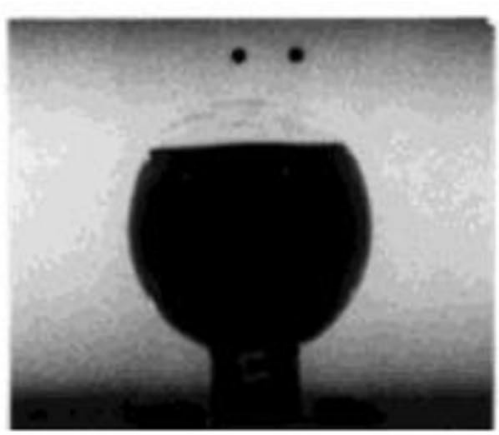

$\approx 0-g$
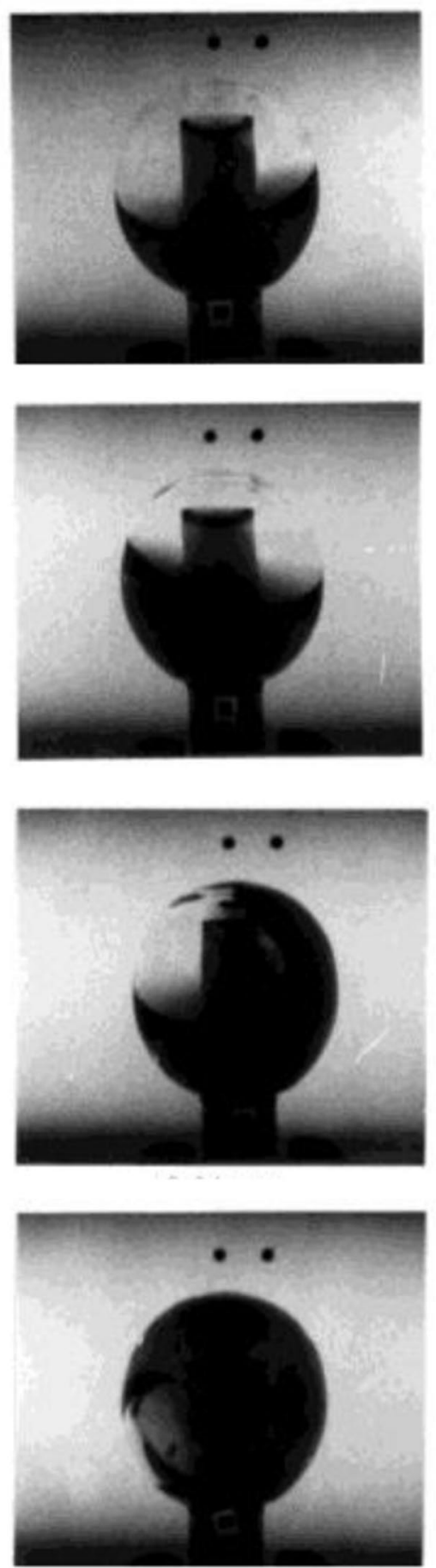

Fig. 6 Original NASA drop tower results of a variety of MA7-like vessels with varying fluid fill fractions [9]. (Courtesy of NASA GRC.)

$$
\begin{aligned}
& x_{1}(u)=a\left(1-\cos u+\int_{0}^{u} \frac{\sin ^{2} \psi}{\sqrt{\sin ^{2} \psi+b^{2} / a^{2}}} \mathrm{~d} \psi\right), \\
& y_{1}(u)=a\left(\sin u+\sqrt{\sin ^{2} u+b^{2} / a^{2}}\right)
\end{aligned}
$$

where $-\pi / 2 \leq u \leq 3 \pi / 2$ for one period of the nodary; and $a$ and $b$ are respectively semimajor and semiminor axes of the hyperbola used to produce the nodary [18] meeting both the inner tank and outer standpipe walls. For a given standpipe, the solutions for parameters $a$ and $b$ are not usually explicit, and a trial-and-error method is used to identify $a$ and $b$ by specifying the location where the nodoid meets the standpipe wall while searching for the proper location where the nodoid meets the tank wall at the prescribed contact angle. As an example, a collection of nodaries for $r=0.333$ is shown in Fig. 15 . Special conditions yield exact solutions, such as when the nodoid meets the tank wall at the tank midplane.

For the fill fraction range producing asymmetric surfaces, the mean curvature of symmetric nodoids was presented in Fig. 13 for $r=0.333$. Note that initially the mean curvature of the asymmetric surface is greater than that of the symmetric nodoid surface, such that a nearly complete shift of the liquid from insideto-outside the standpipe may happen for certain cases, as is observed, for example, in Fig. 16 for $r=0.47$ with slight increase in $\lambda$ from 0.42 to 0.43 . 


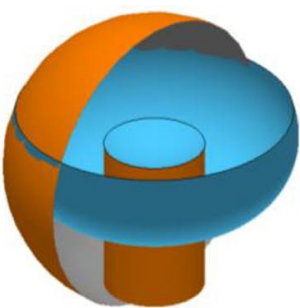

a) $\lambda=0.4$

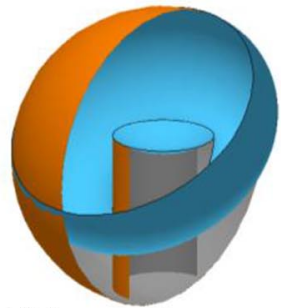

b) 0.5

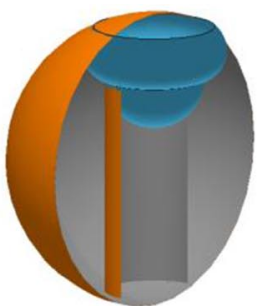

c) 0.92

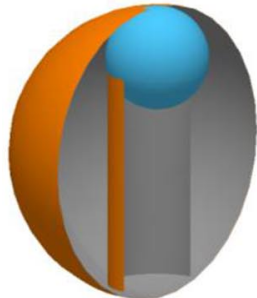

d) 0.958

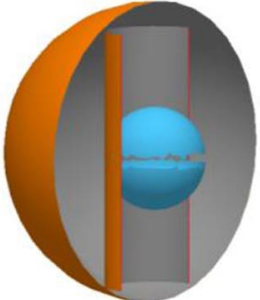

e) 0.962

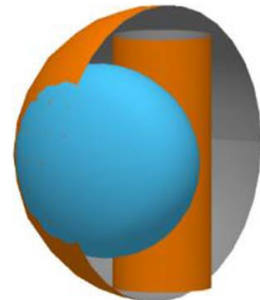

f) 0.81

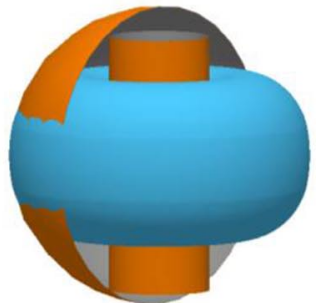

g) 0.4

Fig. 7 Sample computed continuous gas phase equilibria in generalized MA7 geometry: a) symmetric over exit, b) asymmetric over exit, c) symmetric stepped interface, d) bubble over gas standpipe, e) bubble in standpipe, f) asymmetric bubble, and g) symmetric toroid.

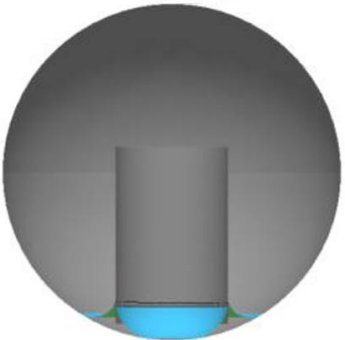

a) $\lambda=0.01$

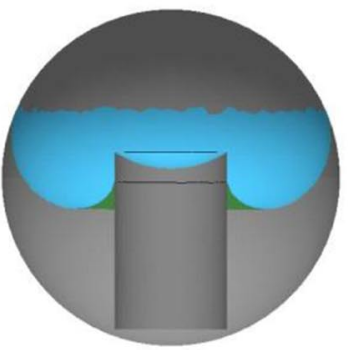

d) 0.47

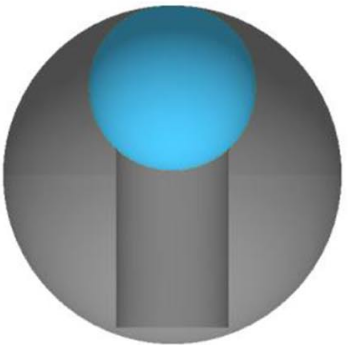

g) 0.88

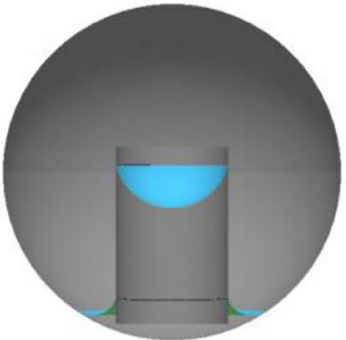

b) 0.08

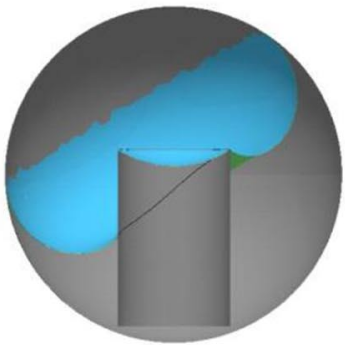

e) 0.48

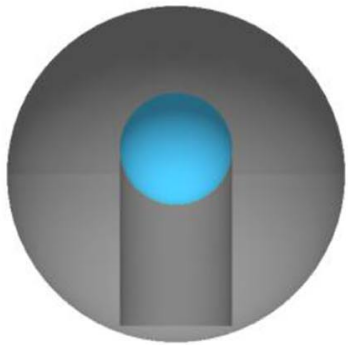

h) 0.963

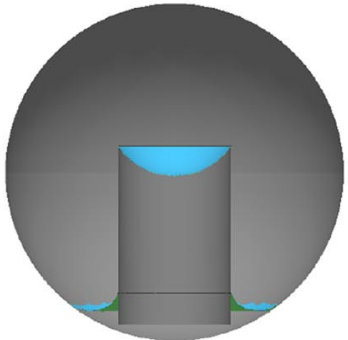

c) 0.1

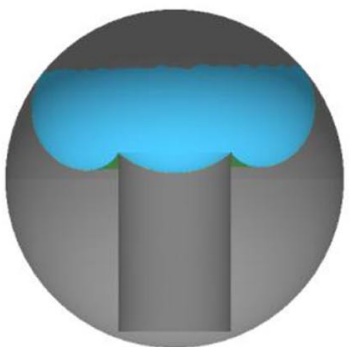

f) 0.65

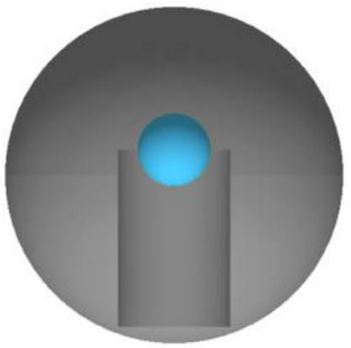

i) 0.99

Fig. 8 Surface configurations with standpipe radius $r=0.333$ and fill fractions $\lambda$ listed, computed using the hybrid surface method. The surface is asymmetric for $\lambda$ between 0.48 and 0.64 .

For any standpipe, the nodoid that meets the pipe at the tank midplane and the standpipe upper outer rim can be determined exactly. The interface inside the standpipe can then be determined from the mean curvature of the nodoid. The volume of the liquid between the entire free surface and the tank wall can then be evaluated and converted to fill fraction $\lambda_{m}$ and $\lambda_{r}$, as presented in Fig. 12 for comparison with the SE-FIT results. A favorable agreement is observed between SE-FIT $\lambda_{l}$ data and nodoid calculations $\lambda_{m}$, indicating that the interface becomes unstable once the contact line on the standpipe exterior wall is near the tank midplane. The limiting fill fraction $\lambda_{r}$ calculated from the nodoids is consistently lower than $\lambda_{u}$ determined numerically, suggesting that the nodoid surface remains unstable even after it meets the standpipe at the upper outer rim.

From the nodoidal surfaces, one can also evaluate the transition point at which the mean curvature of the nodoid is the same as that of 


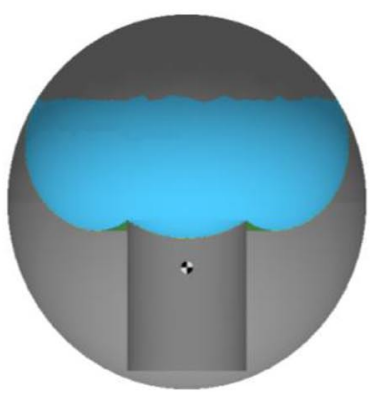

a) $l=0.8$

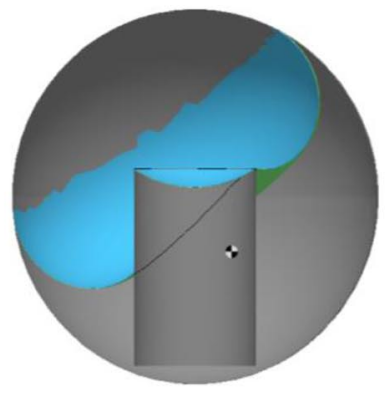

b) 1.052

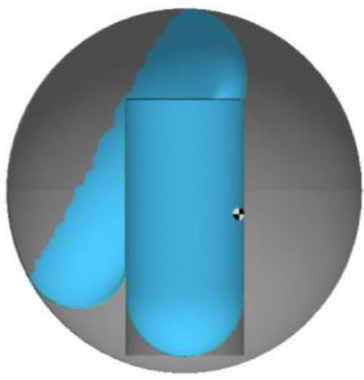

c) 1.4

Fig. 9 Free surface configurations as a function of standpipe length $l$ listed: $r=0.333, \lambda=0.5$.
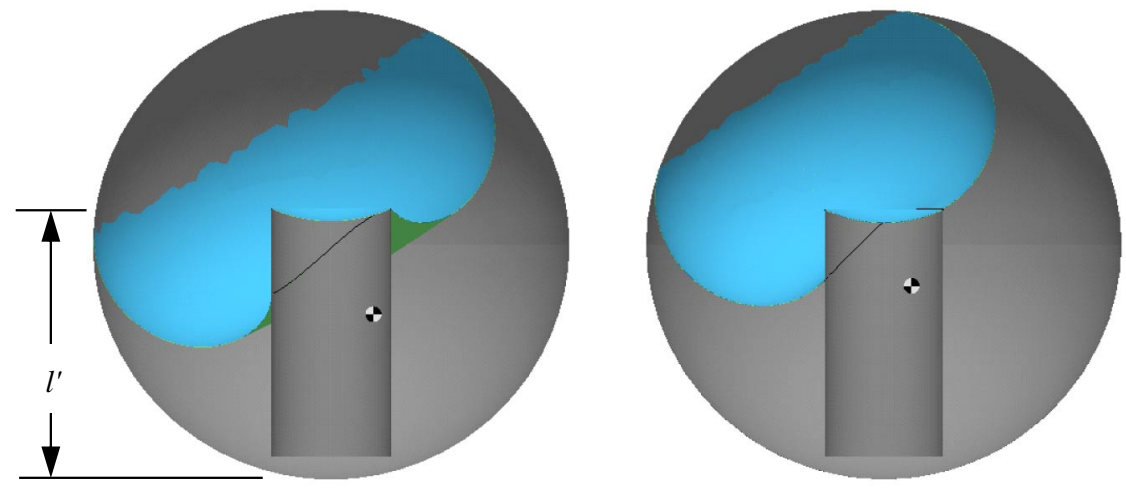

$$
r=0.25, l=1.052, \lambda=0.51
$$

$$
r=0.25, l=1.052, \lambda=0.69
$$
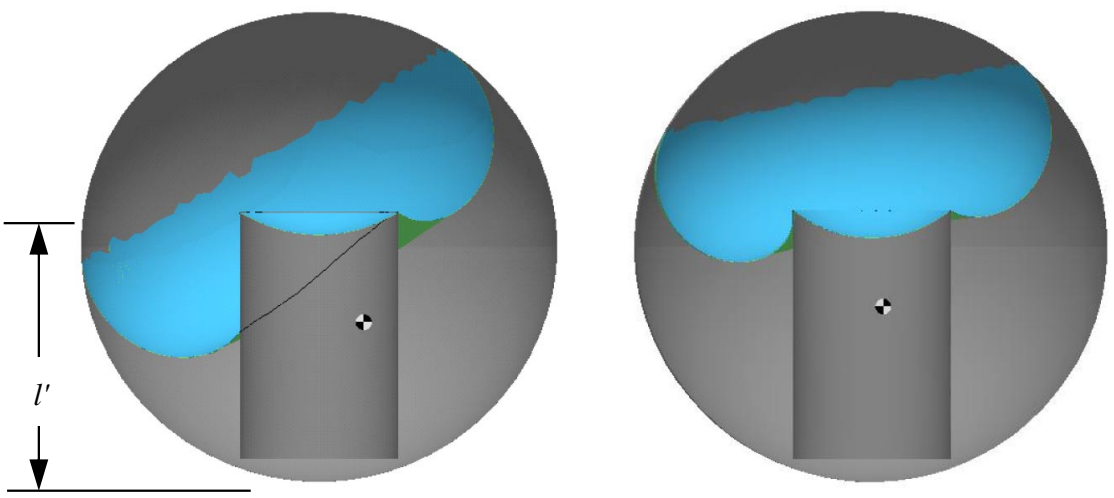

$r=0.333, l=1.052, \lambda=0.48$

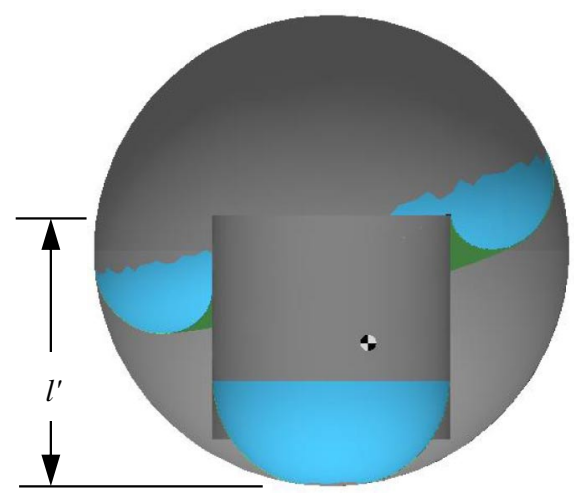

$r=0.5, l=1.052, \lambda=0.27$

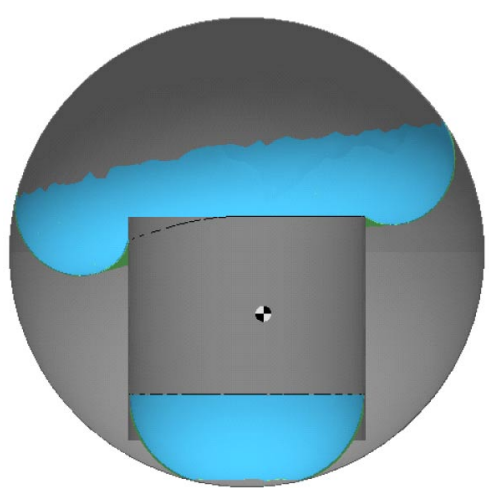

$r=0.5, l=1.052, \lambda=0.40$

Fig. 10 Lower (left column) and upper (right column) fill fraction limit surfaces for computed asymmetric equilibrium interfaces using the standpipe shift perturbation for $r, l$, and $l^{\prime}=1$.15. Mass centers indicated by symbol.

the spherical cap inside the standpipe at $\lambda_{l}$ and $\lambda_{u}$. It can be shown that such a transition occurs at $r \approx 0.493$ for $\lambda_{l}$ and $r \approx 0.471$ for $\lambda_{u}$, which confirms observations of the sudden shift of $\lambda_{l}$ and $\lambda_{u}$ near $r \approx 0.5$ found numerically, as observed in Fig. 12. In addition, it is of interest to point out that the prediction of the surface configuration is complicated for standpipe radii $0.471 \leq r \leq 0.493$. Note that when the free surface 


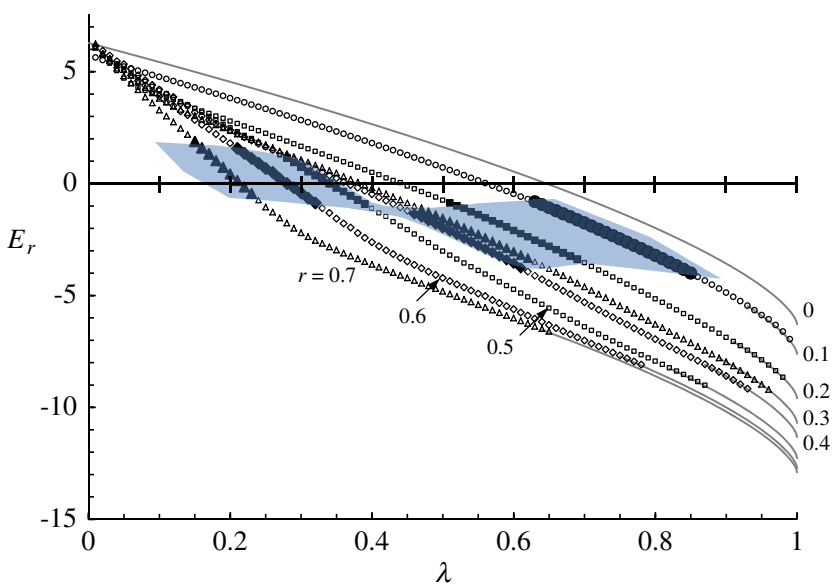

Fig. $11 E_{r}$ computed as function of $\lambda$ for $r$ with $\theta=0 \operatorname{deg}(R=1$, $g=0$ ). Open symbols symmetric surfaces, larger solid symbols asymmetric surfaces, solid grey lines spherical bubble analytic solutions.

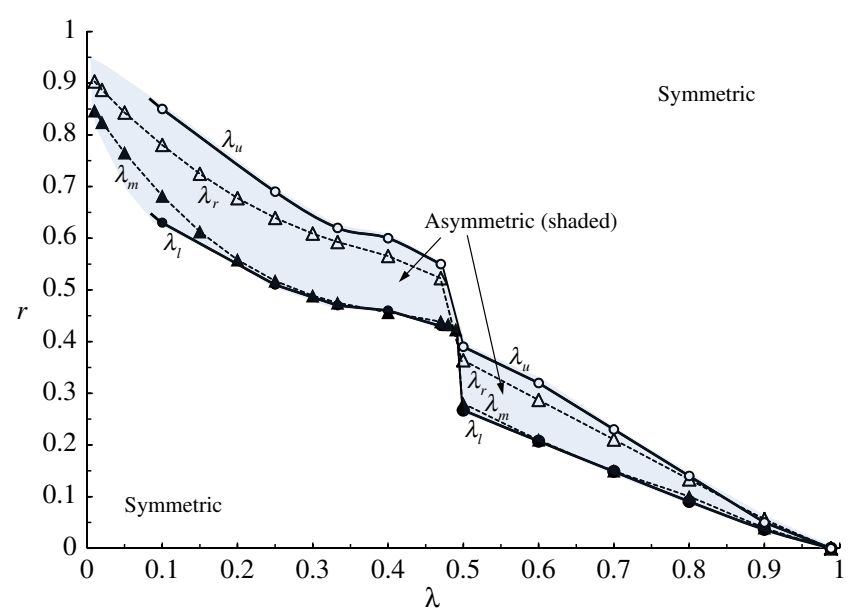

Fig. 12 Asymmetric surface $\lambda$ ranges: circles upper $\lambda_{u}$ and lower $\lambda_{l}$ asymmetric-over-exit results, closed triangles nodoid surfaces meeting the standpipe outer wall at mid-plane $\lambda_{m}$, open triangles nodoid surfaces meeting standpipe outer wall at $\theta=0 \mathrm{deg}$ and upper outer $\operatorname{rim} \lambda_{r}$.

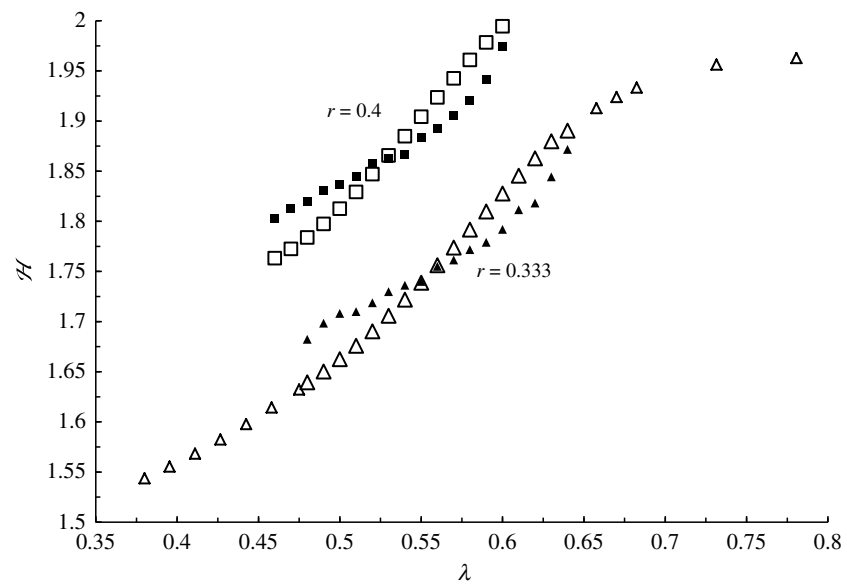

Fig. 13 Mean curvature $\mathcal{H}$ of symmetric surfaces (nodoids, open symbols) and numerically computed (closed) asymmetric surfaces as a function of fill fraction $\lambda$ for $r=4$ (squares) and $r=0.333$ (triangles).

inside the standpipe is near the standpipe lower inner rim, the surface is also part of a nodoid, which is not evaluated deliberately in this study but is assumed to be an extension of the standpipe inner wall only, providing further discrepancies in the comparison. The fill fraction

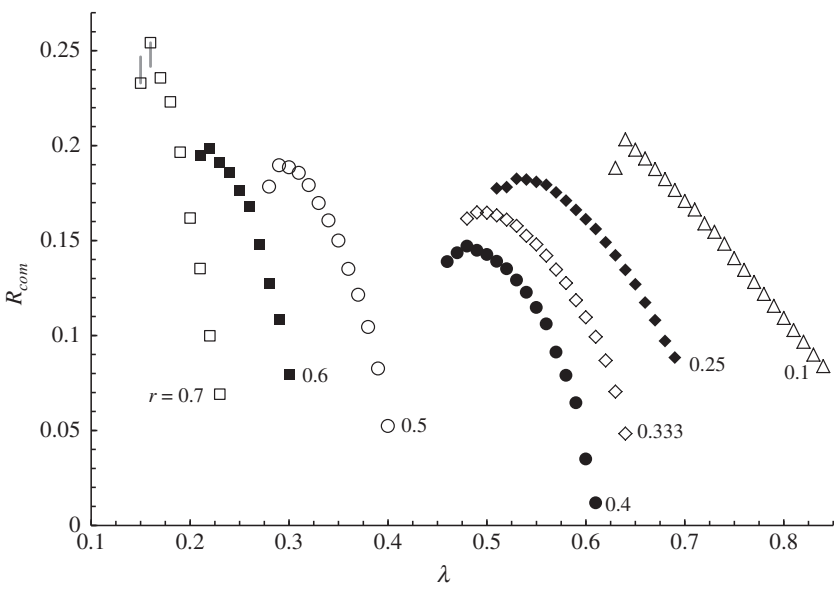

Fig. 14 Radial shift $R_{\text {com }}$ of center of mass for 'asymmetric-over-outlet' surfaces for $\lambda$ and $r$. Uncertainties in numerical data for $r=0.7$ at $\lambda=0.15$ and 0.16 identified by vertical bars.

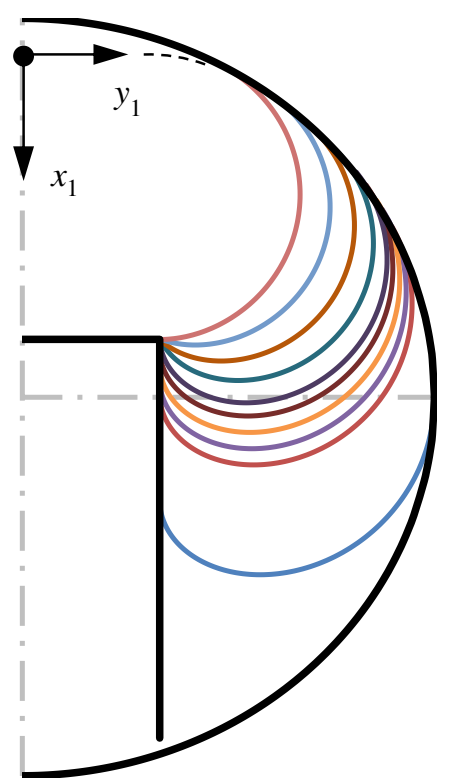

Fig. 15 Nodaries identified with contact line on the standpipe for $r=0.333$.

lower and upper bound data provide useful hints in understanding the instability mechanism of the symmetric nodoidal surfaces in the MA7 geometry. Further investigation along this line is certainly of interest but is not pursued in this study.

The impact of contact angle can be dramatic on the surface configurations, and we provide a single asymmetric surface example for the case of $\theta=90 \mathrm{deg}$ in Fig. 17. For identical conditions, both surfaces are asymmetric with $\lambda=0.51$, with the only difference being a numerical parameter called "gap_constant," which is implemented in the Surface Evolver algorithm for convex walls. The gap_constant value is set to 10 for all numerical data presented in this study as a relatively large value establishing an even distribution of vertices along the contact lines to assure a fast overdamped convergence. We note that Brakke [] recommends gap_constant $=1$. The higher the value of gap_constant, the higher the "gap energy" (gap_quant) error contribution of the contact line region to the overall surface energy. In the case of Fig. 17b, gap_constant is set to the underdamped value of 1 . The specific values of gap_constant, reduced surface energy $E_{r}$, and gap energy gap_quant are included in the caption. Clearly, the value chosen for gap_constant can have a significant impact for large contact angles in axisymmetric containers, which should not be ignored in more 

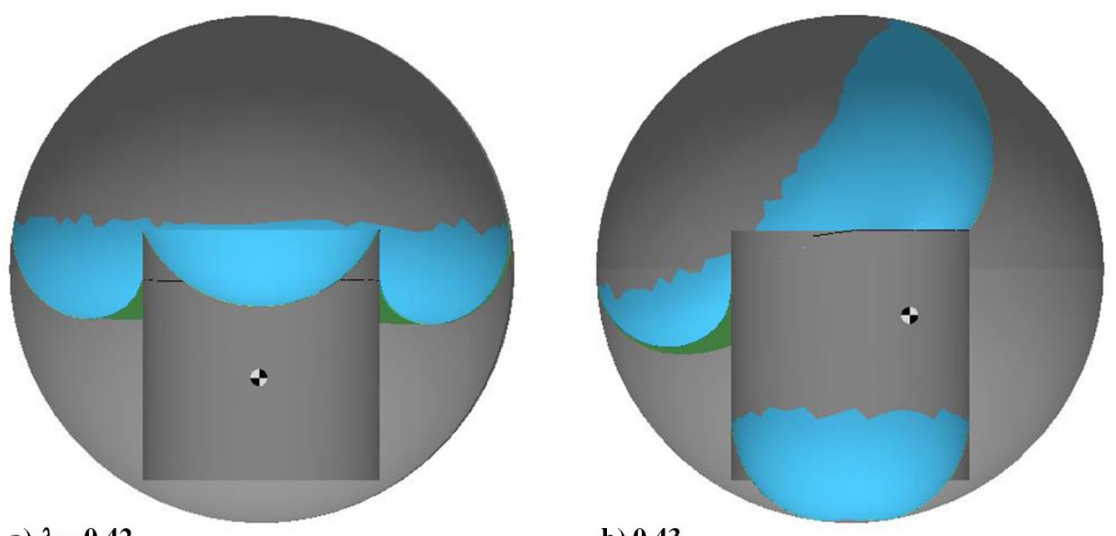

a) $\lambda=0.42$

b) 0.43

Fig. 16 Surface configuration for standpipe radius $r=0.47, l^{\prime}=1.052$. The meniscus inside the standpipe descends toward the tank wall when the surface becomes asymmetric.
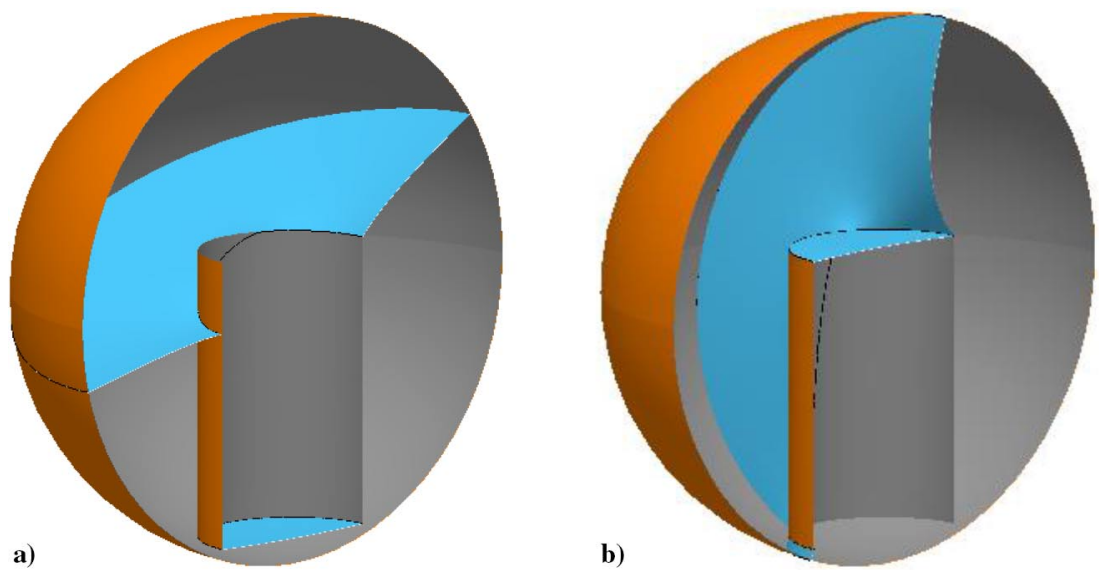

Fig. 17 Sectional views of asymmetric surfaces with $\theta=90 \mathrm{deg}, r=0.333$, and $\lambda=0.51$ : a) gap_constant $=10$, $E_{r}=3.072$, and gap_quant $=0.00637$. b) gap_constant $=1, E_{r}=2.799$, and gap_quant $=0.000438$.

exhaustive analyses. In this example, due largely to changes in gap_quant, the ratio gap_quant $/ E_{r}$ differs by an order of magnitude. The fact that $E_{r}=2.799$ for gap_constant $=1$ is lower than $E_{r}=3.072$ for gap_constant $=10$ suggests that the former is more likely the true equilibrium configuration. We observe that both $E_{r}$ and gap_quant increase with gap_constant. We further conjecture that when gap_constant $=10$, the contribution of gap_quant is likely too large to fully evolve the surface. Deeper investigations of this sort are straightforward and warranted for critical applications.

\section{Discussion}

When employing the contact line method, the geometric perturbation of shifting and recentering the standpipe provides an easy pathway to identify asymmetric equilibrium surfaces. Though not shown here, we also demonstrate how shifting the center of mass of the gas or liquid can accomplish the same results. For the MA7 geometry, the hybrid surface method, however, is effective in identifying the asymmetric surfaces without a perturbation at all when standpipe radius $r \geq 0.4$. The standpipe displacement or center-of-mass shift methods are used when $r<0.4$. A fluid interface tool providing these options can accelerate identification of local asymmetric energy minimizing surfaces in symmetric containers. The routine prediction of global minimizing surfaces remains a prized capability.

Because when using the hybrid surface method, we find that no perturbations are necessary to compute asymmetric equilibrium surfaces, we suspect that SE-FIT results using the contact line method introduce slight energy barriers between unstable symmetric configurations and the slightly lower energy stable asymmetric configurations. During the iterations, SE-FIT monitors the variation of the total energy and performs mesh grooming functions to assure the convergence of the computation. Using the contact line method, for a certain fill fraction range, it is observed that the mesh grooming operation actually assists the free surface in overcoming the artificial energy barrier and establishing asymmetric configurations.

It is clear that the asymmetric surfaces possess asymmetric contact line pinning conditions along the standpipe upper outer rim, insinuating that the former assures the latter. Conceptually, asymmetric surfaces might be expected where local perturbations create higher curvature (lower pressure) regions demanding fluid from lower curvature (higher pressure) regions. But variable contact angle pinning states along the edges of geometric components such as the standpipe upper rim assure that asymmetries can attain equilibrium. An exhaustive numerical study is not presented here, but this brief hindsight exploration of the MA7 experiments quickly reveals the following primary observations.

1) In the manner of MA7, the interface is symmetric and stable when the contact line on the exterior standpipe surface is below the spherical container midplane (Fig. 7a).

2) However, and for the example of the MA7 geometry with an increase in fill fraction from 20 to $48 \%$, when the symmetrical free surface contact line on the standpipe exterior surface is above the container midplane, the surface becomes unstable and forms an asymmetric surface with nonuniform contact angle along a portion of the standpipe upper outer rim (Fig. $7 \mathrm{~b}$ ). 
3) Interfaces are also symmetric and stable when the contact line is pinned along the entire upper outer edge of the standpipe (Figs. $7 \mathrm{c}$ and $7 \mathrm{~d}$ ).

4) Long standpipe lengths can lead to other asymmetric as well as symmetric surface topologies (i.e., Figs. $\underline{7 \mathrm{f}}$ and $\underline{7 \mathrm{~g}}$, respectively).

\section{Conclusions}

Within minutes, the SE-FIT computations are capable of identifying that the MA7 experiment geometry was wisely selected to passively position the liquid for the given fill fraction (Fig. 3). However, for increased liquid fill fractions, longer standpipes, and other geometric variations, multiple interface shapes, including asymmetric surface with large shifts in mass center, are almost certain to arise that are not necessarily desirable from a passive control perspective. With the ability to sweep through changes in container geometry, 50-plus years of continued progress in modeling tools has dramatically added to the ability to design systems that control fluid locations by purely capillary forces that are intricately linked to container shape and fluid-wall wettability.

For over 50 years, the terrestrial understanding of capillarity has been applied to the microgravity conditions often present aboard spacecraft. Since the first fluids experiment aboard the MercuryAtlas 7 mission, the ability to predict such phenomena with increasing ease and precision for systems of increasing complexity is enabling designs to consider exploitation of the capillary force to greater extent. The ability for control of large and small inventories aboard spacecraft using the passive forces of surface tension and clever choice of system geometry beckons the continued pursuit of research and technology development along these lines. At a fundamental level, such work produces designs that avoid failure due to unexpected capillary instability and metastability. It also establishes methods of design that either provides at least backup, or at best primary, means for fluids transport and positioning in otherwise mechanically pumped systems.

\section{Appendix: Reduced Energy}

The surface energy is $E=\sigma_{\mathrm{vs}} A_{\mathrm{vs}}+\sigma_{\mathrm{vl}} A_{\mathrm{vl}}+\sigma_{\mathrm{ls}} A_{\mathrm{ls}}$, where subscript $v$ denotes vapor, $s$ is solid wall, and $l$ is liquid, as identified in Fig. A1. The total area of the wall is $A_{\mathrm{s}}=A_{\mathrm{vs}}+A_{1 \mathrm{~s}}$. Young's equation relating surface tensions and contact angle is $\sigma_{\mathrm{vs}}=\sigma_{\mathrm{vl}} \cos \theta+\sigma_{\mathrm{ls}}$. Substitution of these quantities yields $=\sigma_{\mathrm{vs}} A_{\mathrm{s}}$ $+\sigma_{\mathrm{vl}}\left(A_{\mathrm{vl}}-A_{\mathrm{ls}} \cos \theta\right)$, where $\sigma_{\mathrm{vs}} A_{\mathrm{s}}$ is a constant that does not need to be included in the energy formulations, giving rise to the meaningful surface energy quantity $E_{1}=\sigma_{\mathrm{vl}}\left(A_{\mathrm{vl}}-A_{\mathrm{ls}} \cos \theta\right)$, which, on a per surface tension basis, yields $E_{1} / \sigma_{v l} \equiv E_{2}=A_{\mathrm{vl}}-A_{1 \mathrm{~s}} \cos \theta$. Furthermore, because of the nature of the spherical surface, the integral formulations only calculate the area between contact line and the corresponding equator of the sphere with a negative sign ascribed to the area wherever the contact line lies below the equator. The integration is added to the hemispherical area to yield the true wetted area of the spherical wall. Because the hemispherical area is a constant, it is not included in the energy formulations, which gives rise to the reduced energy:

$$
E_{r}=A_{\mathrm{vl}}-\left(A_{\mathrm{ls}}-A_{h}\right) \cos \theta
$$

where the subscript $h$ denotes hemisphere.

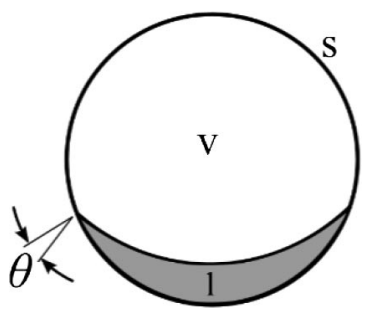

Fig. A1 Schematic of a free surface inside a spherical container.

\section{Acknowledgments}

This work is supported in part through NASA Cooperative Agreements NNX12AO47A and NNX16AC38G. The work was first presented as part of a 50 year review of MA7 as the first fluids experiment in space.

\section{References}

[1] Petrash, D. A., Nussle, R. C., and Otto, E. W., "Effect of the Acceleration Disturbances Encountered in the MA-7 Spacecraft on the Liquid-Vapor Interface in a Baffled Tank During Weightlessness," NASATN D-1577, Jan. 1963.

[2] Reynolds, W. C., "Hydrodynamic Considerations for the Design of Systems for Very Low Gravity Environments," Dept. of Mechanical Engineering, Stanford Univ., TR LG-1, Stanford, CA, 1961.

[3] Concus, P., Finn, R., and Weislogel, M. M., "Capillary Surfaces in an Exotic Container: Results from Space Experiments," Journal of Fluid Mechanics, Vol. 394, Oct. 1999, pp. 119-135. doi: $10.1017 / \mathrm{S} 0022112099005789$

[4] Concus, P., Finn, R., and Weislogel, M. M., "Measurement of Critical Contact Angle in a Microgravity Experiment," Experiments in Fluids, Vol. 28, No. 3, 2000, pp. 197-205. doi: $10.1007 / \mathrm{s} 003480050379$

[5] Weislogel, M. M., Jenson, R. M., Chen, Y., Collicott, S. H., and Williams, S., "Geometry Pumping on Spacecraft: The CFE-Vane Gap Experiments on ISS," Journal of the Japan Society of Microgravity Application, Vol. 25, No. 3, 2008, pp. 291-295.

[6] Chen, Y., Jenson, R. M., Weislogel, M. M., and Collicott, S. H., "Capillary Wetting Analysis of the CFE-Vane Gap Geometry," 46th AIAA Aerospace Sciences Meeting and Exhibit, AIAA Paper 20080817, Jan. 2008.

[7] Chen, Y., Schaeffer, B. M., Weislogel, M. M., and Zimmerli, G. A., "Introducing SE-FIT: Surface Evolver-Fluid Interface Tool for Studying Capillary Surfaces," 49th AIAA Aerospace Sciences Meeting, AIAA Paper 2011-1319, Jan. 2011.

[8] Brakke, K. A., "The Surface Evolver," Experimental Mathematics, Vol. 1, No. 2, 1992, pp. 141-165.

[9] Petrash, D. A., Nelson, T. M., and Otto, E. W., "Effect of Surface Energy on the Liquid-Vapor Interface," NASA TN D-1582, Jan. 1963.

[10] Hung, R. J., Tsao, Y. D., and Hong, B. B., "Bubble Behaviors in a Slowly Rotating Helium Dewar in a Gravity Probe-B Spacecraft Experiment," Journal of Spacecraft, Vol. 26, No. 3, 1989, pp. 167-172. doi: $10.2514 / 3.26049$

[11] Bayt, R. L., and Collicott, S. H., "Effects of an Elliptic End-Cap on the Ullage Bubble Stability in the Gravity Probe-B Satellite," 34th AIAA Aerospace Sciences Meeting and Exhibit, AIAA Paper 1996-0596, Jan. 1996.

[12] Chen, Y., and Collicott, S. H., "Effects of Wicking and Spin on Bubble Position in the Gravity Probe-B Helium Tank Geometry," 40th AIAA Aerospace Sciences Meeting and Exhibit, AIAA Paper 2002-1004, Jan. 2002.

[13] Collicott, S. H., "Example Impact of Nonuniform Acceleration Fields on Liquids in Spacecraft," Journal of Spacecraft and Rockets, Vol. 44, No. 3, May 2007, pp. 725-727. doi: $10.2514 / 1.28561$

[14] Slobozhanin, L. A., Alexander, J. I. D., Collicott, S. H., and Gonzalez, S. R., "Capillary Pressure of a Liquid in a Layer of Close-Packed Uniform Spheres," Physics of Fluids, Vol. 18, 2006, Paper 082104. doi:10.1063/1.2236123

[15] Collicott, S. H., and Weislogel, M. M., "Computing Existence and Stability of Capillary Surfaces Using Surface Evolver," AIAA Journal, Vol. 42, No. 2, 2004, pp. 289-295. doi: 10.2514/1.9093

[16] Seebold, J. G., Hollister, M. P., and Satterlee, H. M., "Capillary Hydrostatics in Annular Tanks," Journal of Spacecraft, Vol. 4, No. 1, Jan. 1967, pp. 101-105.

[17] Langbein, D., Capillary Surfaces: Shape-Stability-Dynamics, in Particular Under Weightlessness, Springer Tracts in Modern Physics, Springer, Heidelberg, 2002, p. 178.

[18] Ćirić, M., "Notes on Constant Mean Curvature Surfaces and Their Graphical Presentation," Filomat, Vol. 23, No. 2, 2009, pp. 97-107. 